\title{
BRADLEY-TERRY MODEL FOR ASSESSING THE PERFORMANCE OF TEN ODI CRICKET TEAMS ADJUSTING FOR HOME GROUND EFFECT
}

\author{
Md. Mazharul Islam ${ }^{1}$, Jahidur Rahman Khan ${ }^{1}$, Enayetur Raheem ${ }^{2}$ \\ ${ }^{1}$ Institute of Statistical Research and Training, University of Dhaka \\ ${ }^{2}$ Biomedical Research Foundation (BRF), Dhaka, Bangladesh
}

\begin{abstract}
The analysis of sports data, especially cricket is an interesting field for the statisticians. Every year, a large number of cricket tournaments take place among the cricket playing nations. It is of interest to study their performance when they play with each other in a one-day international (ODI) match or a test match. In this study, we assess the performance of top ten cricket teams in the ODI cricket match and make a comparison among them. The abilities of teams change over time. As a result, not a single team dominates the game over a long period. Therefore, a paired comparison method is more reliable and appropriate to compare more than two teams at the same time based on the outcomes of the matches they play. Arguably, a team's performance also depends on whether they play at home or away. In this study, we consider Bradley-Terry model, a widely accepted model for pairwise comparison. In that, we consider home and away effect to demonstrate how the home advantages differ among these teams.
\end{abstract}

Key words: Pairwise comparison, measuring performance in Cricket, home and away effect.

\section{Introduction}

The game of cricket is popular in many countries and is getting even more popularity day-by-day. During 2011 and 2015, a total of 981 cricket matches have been played in different formats: 210 test matches, 493 ODI matches, and 278 T-20 matches [14]. The International Cricket Council (ICC) has 106 members: 10 full members, 39 associate members and 56 affiliate members [14]. The number of cricket playing countries varies in ODI and T-20 formats (all full members plus a few members from the associate and affiliate members), although only the countries with full-membership play test cricket. Ranking the member countries is an interesting and ongoing process. It is of great importance to the fans to compare among the teams particularly ahead of a multi-nation tournament. Normally, a team with a higher rank gets somewhat more attention. However, upset happens as a team with a higher rank may not necessarily win over a lower ranked team. Arguably, in a multi-nation tournament, a team with better performance in the recent past compared to others is more likely to perform better in the competition such as reaching the quarter-final or semi-final stages. This likelihood may affect supporters, sponsors, as well as team management towards the selection of players for a particular game in the tournament. 
Cricket is played by two teams with 11 players each. The game is divided into two phases: batting and bowling. A toss decides which team would bat first or bowl first. Performance can be measured at the individual level and at the team level over the two phases of a match. Gweshe and Durbach (2013) studied the efficiency of individual player performance at 2011 world cup cricket using a combined data envelopment analysis (DEA) and stochastic multi criteria acceptability analysis (SMAA) approach where players specific inputs such as balls faced by the batsmen and balls bowled by the bowlers are converted outputs indicative of performance. Substantial discrepancies were identified between the players' rank ordering by efficiency and by traditional absolute measures of performance [12]. Moreover, they found the combination of DEA and SMAA methods appears to be a useful technique to measure the efficiency of cricket players in the limited over game. Lewis (2008) provided ranking among players based on individual batting, bowling and all-round (both batting and bowling) performances in ODI cricket. Predicting match outcomes and identifying factors associated with it are often of interest that have been found in the literature. Louzada et al. (2014) considered a linear model to predict match outcomes in the English Premier League. Cerqueira et al. (2017) proposed a latent causal model to measure the team quality and its correlates. However, the performance of a cricket team is measured by the outcome of a match - win, loss or tie. The outcome of a cricket match depends on a number of known and unknown factors. It has been found in the literature that apart from the strength of each team, the strength of the opponent, the venue, toss and a number of other factors may influence the result of a match ([9], [10], [4]). Clarke (1988) studied optimal scoring rates using dynamic programming methods and suggested that teams would be better served by scoring at higher rates early in the innings. de Silva and Swartz (1997) showed that toss of a match provides no competitive advantage, whereas home advantage increases the probability of winning of a team in ODI cricket. Bailey and Clarke (2006) explored the impact of different factors on the outcome of ODI cricket matches such as a home ground advantage, team quality, and current form of the players. Allsopp and Clarke (2004) measured and compared the strength of teams in terms of overall ratings, which is obtained by combining batting and bowling ratings of the respective teams, for ODI and test matches for the period of 1997 to 2001 using multiple linear regression techniques.

Method of paired comparisons (PC) is used often to rank different sports teams, where preference among teams is often the main objective of interest. PC is a well-established method, where individuals are asked to repeatedly judge which of the pairwise objects they prefer on the basis of evaluation [21]. The PC data are expressed as a preference matrix, which is analyzed through PC models. Thurstone (1927) studied that the outcomes of the pairwise comparison follow a normal distribution, but Bradley (1953) assumed logistic distribution to present PC models. Stern (1990a, 1990b) proposed the gamma models for PC. Thurstone-Mosteller model for PC was used to analyze volleyball data [7], Stern used a PC model to analyze sports datasets for the National League baseball season, and football data were analyzed by using Bradley-Terry model ([7], [20], [13]). Neil and Jonathan (2015) investigated the use of Bradley-Terry models to analyze test match cricket, Abbas and Aslam (2009) showed that any group of individuals may be ranked using the Cauchy PC model via a Bayesian approach with 
an application on five top-ranked ODI cricket teams. Stern (2011) explored generalized Bradley-Terry model for assessing the performance of ODI cricket teams.

Several studies considered the PC model to accommodate ties and home-ground effects on sports outcomes ([19], [11], [14], [16]). A possible implication of the team's ability is to rank these teams according to their performances in completed matches. For the analysis of such PC data, we refer to the well-known Bradley-Terry (BT) model [6]. Agresti (2014) compared performances of US basketball teams using this model. The BT model is widely accepted to provide an overall assessment of the performance of a group of individuals using pairwise comparison [6]. This model quantifies the level of preference of an individual over others with a view to organizing them in accordance with their performances. Moreover, prediction of the results of future comparisons can also be depicted from this logit type model ([19], [24]). Although PC models are widely applicable in sports data, these models were used in a fewer number of studies to analyze cricket matches. In this paper, we consider ODI matches of top ten teams in ICC ranking and explore their performance using the BT model with adjusting for home ground effect.

The remaining part of the article is organized as follows. In next Section 2, we discuss the formulation of BT model and the estimation of model parameters. In Results section 3, we summarize the findings of this study. Overall discussion and conclusion are presented in last section 4.

\section{Data and Methodology}

We focus on studying the ODI matches played between January, 2010 to August, 2015. All matches played during this time window were considered except tied and suspended matches. Data were retrieved from ESPN Cricinfo (website link) on ODI matches played by the top ten teams (Australia, South Africa, New Zealand, India, England, Sri Lanka, Pakistan, West Indies, Bangladesh, and Zimbabwe). Data contains the outcome of the match (win/loss), the home team, the away team, ground the match was played, and other match related information such as margin of win (by how many runs or wickets), winning toss (head/tail) and match date.

\section{Model}

In the present study, we consider a pairwise comparison of cricket teams. In the game of cricket, a team can play with only one of the other teams in a single game. Each team consists of eleven players; yet by pairwise comparison, we mean comparison between a pair of team; not between pairwise comparisons between individual players. In this paper, an overall ability among these teams based on match results from pairwise contests has been depicted.

\section{Bradley-Terry Model}

Consider a set of $k$ teams playing with each other. We wish to know the probability that team $i$ will beat team $j$ assuming that a tie cannot occur. BT model can be used to calculate the probability as

$$
p_{i j}=P(\text { team } i \text { beats team } j)=\frac{\pi_{i}}{\pi_{i}+\pi_{j}}
$$


where, $p_{i j}+p_{j i}=1$ for all pairs, $\pi_{i}$ represents the skill or ability of team $i$, calculated as the proportion of all matches won by team $i$ against all the other teams. Now it follows from [2] that

$$
\log \frac{p_{i j}}{p_{j i}}=\gamma_{i}-\gamma_{j}
$$

where $\gamma_{i}=\log \pi_{i}$ and $\gamma_{j}=\log \pi_{j}$.

The restriction imposed over $p_{i j}$ turns equation (2) into a logit model. It follows from equation 2 that

$$
\log \frac{p_{i j}}{1-p_{i j}}=\gamma_{i}-\gamma_{j}
$$

In many real life experiments involving PC, there is often a factor that, independent of the attributes of the respective entities, influences the outcome of the experiment. Arguably, a team may get some extra advantages if it plays at its home ground. The advantage could be the familiar environment, supporters and many others. BT approach can consider these factors into the model. Let $\alpha>0$ be the advantage gained by team $i$ from the external effect (e.g., home advantage). Then we may write (3) as

$$
\log \frac{p_{i j}^{*}}{1-p_{i j}^{*}}=\gamma_{i}-\gamma_{j}+\alpha
$$

where, $p_{i j}^{*}$ denotes probability that team $i$ beats team $j$ in team $i$ 's home ground.

\subsection{Estimation}

If each pair of teams $i$ and $j$ play $n_{i j}$ games against each other with team $i$ winning $y_{i j}$ of them, and all games are assumed independent, the likelihood function is

$$
L(\boldsymbol{\pi})=\prod_{i<j} \frac{\pi_{i}^{y_{i j}} \pi_{j}^{n_{i j}-y_{i j}}}{\left(\pi_{i}+\pi_{j}\right)^{n_{i j}}}
$$

This can be simplified to

$$
L(\boldsymbol{\pi})=\frac{\prod_{i} \pi_{i}^{y_{i}}}{\prod_{i<j}\left(\pi_{i}+\pi_{j}\right)^{n_{i j}}} .
$$

After adjusting home ground effect, the likelihood function will take the form

$$
L(\boldsymbol{\pi})=\prod_{i<j} \frac{e^{n^{i j} \alpha} \pi_{i}^{y_{i j}} \pi_{j}^{n_{i j}-y_{i j}}}{\left(e^{\alpha} \pi_{i}+\pi_{j}\right)^{y_{i j}}\left(e^{\alpha} \pi_{j}+\pi_{i}\right)^{n_{i j-} y_{i j}}} .
$$

By using maximum likelihood and penalized quasi-likelihood (for random effect model) estimation with iteratively reweighted least squares we can obtain $\hat{\pi}$. 


\section{Results}

Table 1 shows the summary of ODI matches of top ten cricket teams. Column 1 shows the team, column 2 shows the number of games played by the team, column 3 shows the percentage of games owned when played at the home ground, column 4 shows the percentage of games owned when played at other ground, column 5 shows the percentage of games lost when played at the home ground and column 6 shows the percentage of games lost when played at other ground. Sri Lanka played maximum matches (147 matches) among all other teams where Zimbabwe played 71 matches. Pakistan played only 2 matches at their home because of security problems. Australia won about $40 \%$ of their matches at home condition where about $24 \%$ in away condition. India lost about $28 \%$ of their matches in outside of their home, which is 4 times greater than losing at their home condition.

Table 1: Summary of ODI cricket matches of top ten cricket teams between January, 2010 and August, 2015

\begin{tabular}{lccccc}
\hline \multicolumn{1}{c}{ Team } & Played & Home win (\%) & Away win (\%) & Home lose (\%) & Away lose (\%) \\
\hline Australia & 114 & 40.35 & 24.56 & 10.53 & 24.56 \\
England & 111 & 29.73 & 18.92 & 20.72 & 30.63 \\
India & 140 & 26.43 & 37.14 & 7.86 & 28.57 \\
New Zealand & 101 & 28.71 & 18.81 & 16.83 & 35.64 \\
Pakistan & 122 & 1.64 & 45.08 & 0.00 & 53.28 \\
South Africa & 99 & 19.19 & 41.41 & 13.13 & 26.26 \\
Sri Lanka & 147 & 24.49 & 28.57 & 14.97 & 31.97 \\
West Indies & 99 & 18.18 & 20.20 & 21.21 & 40.40 \\
Bangladesh & 88 & 32.95 & 6.82 & 37.50 & 22.73 \\
Zimbabwe & 71 & 15.49 & 2.82 & 38.03 & 43.66 \\
\hline
\end{tabular}

A simple logistic regression of teams' performances (win $=1$, lose $=0$ ) with covariate of home advantage (played at home $=1$, away $=0$ ) suggests that the most of the teams' performance significantly differs with home and away condition (result not presented in this paper). Overall, we found that a team is more likely to win if it plays at its own home ground. Crude odds ratios (CORs) from simple logistic regressions fit: Australia 3.83, England 2.32, India 2.59, New Zealand 3.23, Sri Lanka 1.83, South Africa 0.93, West Indies 1.71, Bangladesh 2.93 and 
Zimbabwe 6.31.COR is very high (exp (15.73)) for Pakistan because only 2 matches were played in home ground.

Therefore, two different BT models were considered; one with the overall ability among these teams, and the other after adjusting for home effects (Table 2). We also tested for association between winning the toss and winning the match but did not find any statistically significant association.

Table 2 shows the coefficients of fitted BT models of two scenarios. Matches resulted in ties or no results were excluded from the analysis. We arbitrarily considered "West Indies" as our reference category. Exponential of the fitted coefficient implies preference or superiority of the team with respect to the reference team (West Indies). Team Australia was found to have the maximum ability among all other teams with an estimated coefficient of 1.18 . The exponent of this estimate is 3.28 for team Australia to team West Indies informs us that the Australia is ranked significantly higher by over 3 times compare to the West Indies. Our results show that Australia, England, India, South Africa, and Sri Lanka significantly dominate over West Indies. Moreover, the ratio for any two teams can be calculated by taking exponential of the difference between the corresponding teams' parameter estimates; e.g. Australia is slightly more likely to defeat England $\left(\mathrm{e}^{(1.18-0.63)}=1.73\right.$ times $)$ than the reverse, that means Australia is ranked higher than England.

Table 2: Estimated of parameters of Bradley-Terry models

\begin{tabular}{lcccccccc}
\hline & \multicolumn{3}{c}{ Model 1 } & \multicolumn{5}{c}{ Model 2 } \\
\hline Team & $\gamma$ & S.E. & $\boldsymbol{e}^{\gamma}$ & $95 \%$ CI & $\gamma$ & S.E. & $\boldsymbol{e}^{\gamma}$ & $95 \%$ CI \\
Australia & $1.18^{* * *}$ & 0.29 & 3.25 & $1.84-5.75$ & $1.18^{* * *}$ & 0.29 & 3.25 & $1.84-5.75$ \\
Bangladesh & -0.21 & 0.30 & 0.81 & $0.45-1.46$ & -0.51 & 0.31 & 0.60 & $0.33-1.10$ \\
England & $0.68^{*}$ & 0.29 & 1.97 & $1.12-3.48$ & $0.63 *$ & 0.30 & 1.88 & $1.04-3.38$ \\
India & $1.01^{* * *}$ & 0.27 & 2.74 & $1.62-4.66$ & $1.07 * * *$ & 0.28 & 2.93 & $1.68-5.05$ \\
New Zealand & 0.33 & 0.29 & 1.39 & $0.79-2.46$ & 0.32 & 0.29 & 1.37 & $0.78-2.43$ \\
Pakistan & 0.38 & 0.28 & 1.46 & $0.84-2.53$ & $0.69 *$ & 0.29 & 1.99 & $1.13-3.52$ \\
South Africa & $0.89 * *$ & 0.29 & 2.44 & $1.38-4.30$ & $1.03 * * *$ & 0.30 & 2.79 & $1.56-5.04$ \\
Sri Lanka & $0.75^{* *}$ & 0.27 & 2.12 & $1.25-3.59$ & $0.79 * *$ & 0.29 & 2.21 & $1.25-3.89$ \\
Zimbabwe & $-1.17 * *$ & 0.37 & 0.31 & $0.15-0.64$ & $-1.37 * * *$ & 0.38 & 0.26 & $0.12-0.54$ \\
West Indies & 0.00 & - & 1.00 & - & 0.00 & - & 1.00 & - \\
Home effect $(\boldsymbol{\alpha})$ & - & - & - & - & $0.61 * * *$ & 0.12 & 1.84 & $1.45-2.33$ \\
\hline
\end{tabular}

S.E. - standard error; CI - confidence interval; $\bullet p<0.10,{ }^{*} \mathrm{p}<0.05, * * \mathrm{p}<0.01, * * * \mathrm{p}<0.001$

From Model-2 (Table 2) we find the home effect to be a significant factor on match result. The estimated coefficient corresponding to home effect is 0.61 (i.e. 1.85 with $95 \%$ CI: 1.45 - 
2.33), which implies an extra benefit in favor of the home team. Model-2 adjusts the home advantage and increases the likelihood of winning the match if they play at their home field.

Table 3 presents the probability of winning a game without adjusting for home effect. The probabilities were calculated from estimated coefficients in table 2 (model -1) and using equation (1). Winning teams are presented alongside at $2^{\text {nd }}$ column, whereas $2^{\text {nd }}$ row of the table presents losing teams. Ranking of the ability of these teams was obtained based on these probabilities. Of all teams, Australia had the winning probability greater than 0.5 against all other teams suggesting their superiority among the cricket playing nations. On the other hand, Zimbabwe was placed at the bottom in terms of calculating probabilities of winning against each of the teams. A possible ranking of preference (from the most superior to the least) may be presented as - Australia, India, South Africa, Sri Lanka, England, Pakistan, New Zealand, West Indies, Bangladesh and Zimbabwe.

Table 3: Probability of winning by teams: unadjusted for home ground effect

\begin{tabular}{|c|c|c|c|c|c|c|c|c|c|c|c|}
\hline \multirow{3}{*}{ Team } & & \multirow[b]{2}{*}{ AUS } & \multirow[b]{2}{*}{ BAN } & \multirow[b]{2}{*}{ ENG } & \multirow[b]{2}{*}{ IND } & \multirow[b]{2}{*}{$\mathrm{NZ}$} & \multirow[b]{2}{*}{ PAK } & \multirow[b]{2}{*}{ SA } & \multirow[b]{2}{*}{ SR } & \multicolumn{2}{|c|}{ Loser } \\
\hline & & & & & & & & & & ZIM & WI \\
\hline & & & & & & & & & & & \\
\hline & AUS & - & 0.8017 & 0.6248 & 0.5452 & 0.7014 & 0.6927 & 0.5736 & 0.6077 & 0.9133 & 0.7663 \\
\hline & BAN & 0.1983 & - & 0.2917 & 0.2287 & 0.3676 & 0.3579 & 0.2497 & 0.2770 & 0.7226 & 0.4479 \\
\hline & ENG & 0.3752 & 0.7083 & - & 0.4185 & 0.5852 & 0.5751 & 0.4469 & 0.4819 & 0.8634 & 0.6633 \\
\hline & IND & 0.4548 & 0.7713 & 0.5815 & - & 0.6622 & 0.6528 & 0.5288 & 0.5637 & 0.8978 & 0.7324 \\
\hline \multirow[t]{6}{*}{ Winner } & NZ & 0.2986 & 0.6324 & 0.4148 & 0.3378 & - & 0.4896 & 0.3641 & 0.3973 & 0.8176 & 0.5826 \\
\hline & PAK & 0.3073 & 0.6421 & 0.4249 & 0.3472 & 0.5104 & - & 0.3738 & 0.4073 & 0.8237 & 0.5927 \\
\hline & SA & 0.4264 & 0.7503 & 0.5531 & 0.4712 & 0.6359 & 0.6262 & - & 0.5352 & 0.8867 & 0.7091 \\
\hline & SR & 0.3923 & 0.7230 & 0.5181 & 0.4363 & 0.6027 & 0.5927 & 0.4648 & - & 0.8718 & 0.6792 \\
\hline & ZIM & 0.0867 & 0.2774 & 0.1366 & 0.1022 & 0.1824 & 0.1763 & 0.1133 & 0.1282 & - & 0.2375 \\
\hline & WI & 0.2337 & 0.5521 & 0.3367 & 0.2676 & 0.4174 & 0.4073 & 0.2909 & 0.3208 & 0.7625 & - \\
\hline
\end{tabular}

AUS: Australia, SA: South Africa, NZ: New Zealand, IND: India, ENG: England, SR: Sri Lanka, PAK: Pakistan, WI: West Indies, BAN: Bangladesh, ZIM: Zimbabwe 
In Table 4, the winning probabilities of a team are distinguished by the venue of respective matches. Organization of Table 4 is similar to the previous one. A team would get extra advantages which were captured by adjusting for the home ground effect estimated in Table 2 (model-2). Australia was the only team having the probability of winning a match against remaining others greater than 0.5 at home and abroad. India was more likely (with probability 0.62) to win matches against Australia at its home-ground, but less likely to win (with probability 0.47) abroad. Probabilities of winning by England, Pakistan, South Africa and Sri Lanka against India were greater than 0.5 at their respective home-ground, but they were less than 0.5 at outside of home-ground. In other words, these teams are more likely to win a match played at their home ground than playing at the opponent team's home ground. All of the teams except Bangladesh, West Indies and Zimbabwe had winning probabilities greater than 0.5 against New Zealand in both home and away. Zimbabwe had all the winning probabilities less than 0.5 whether they played at home or abroad.

Table 4: Probability of winning by teams: adjusted for home ground effect

Loser

\begin{tabular}{|c|c|c|c|c|c|c|c|c|c|c|c|}
\hline \multicolumn{2}{|l|}{ Team } & \multicolumn{2}{|c|}{ AUS } & \multicolumn{2}{|c|}{ BAN } & \multicolumn{2}{|c|}{ ENG } & \multicolumn{2}{|c|}{ IND } & \multicolumn{2}{|c|}{$\mathrm{NZ}$} \\
\hline \multicolumn{2}{|c|}{ Venue of winner } & Home & Away & Home & Away & Home & Away & Home & Away & Home & Away \\
\hline & AUS & - & - & 0.9090 & 0.8438 & 0.7613 & 0.6331 & 0.6724 & 0.5261 & 0.8142 & 0.7033 \\
\hline & BAN & 0.2549 & 0.1562 & - & - & 0.3712 & 0.2420 & 0.2753 & 0.1704 & 0.4479 & 0.3049 \\
\hline & ENG & 0.5173 & 0.3669 & 0.8528 & 0.7580 & - & - & 0.5433 & 0.3915 & 0.7176 & 0.5788 \\
\hline & IND & 0.6249 & 0.4739 & 0.9000 & 0.8296 & 0.7419 & 0.6085 & - & - & 0.7979 & 0.6811 \\
\hline \multirow[t]{4}{*}{ Winner } & NZ & 0.4382 & 0.2967 & 0.8082 & 0.6951 & 0.5737 & 0.4212 & 0.4640 & 0.3189 & - & - \\
\hline & PAK & 0.5317 & 0.3804 & 0.8599 & 0.7684 & 0.6620 & 0.5144 & 0.5576 & 0.4053 & 0.7291 & 0.5928 \\
\hline & SA & 0.6132 & 0.4616 & 0.8955 & 0.8225 & 0.7322 & 0.5966 & 0.6376 & 0.4876 & 0.7898 & 0.6702 \\
\hline & SR & 0.5564 & 0.4042 & 0.8714 & 0.7857 & 0.6839 & 0.5392 & 0.5820 & 0.4296 & 0.7483 & 0.6166 \\
\hline
\end{tabular}




\begin{tabular}{l|lllllllllll} 
ZIM & 0.1271 & 0.0730 & 0.4403 & 0.2985 & 0.2007 & 0.1196 & 0.1391 & 0.0804 & 0.2566 & 0.1573 \\
WI & 0.3625 & 0.2352 & 0.7545 & 0.6243 & 0.4952 & 0.3466 & 0.3870 & 0.2545 & 0.5741 & 0.4217 \\
& & & & & & & & & & \\
\hline
\end{tabular}

Loser

\begin{tabular}{|c|c|c|c|c|c|c|c|c|c|c|c|}
\hline \multicolumn{2}{|l|}{ Team } & \multicolumn{2}{|c|}{ PAK } & \multicolumn{2}{|c|}{ SA } & \multicolumn{2}{|c|}{ SR } & \multicolumn{2}{|c|}{ ZIM } & \multicolumn{2}{|c|}{ WI } \\
\hline \multicolumn{2}{|c|}{ Venue of winner } & Home & Away & Home & Away & Home & Away & Home & Away & Home & Away \\
\hline & AUS & 0.7507 & 0.6196 & 0.6832 & 0.5384 & 0.7316 & 0.5958 & 0.9592 & 0.9270 & 0.8574 & 0.7648 \\
\hline & BAN & 0.3578 & 0.2316 & 0.2853 & 0.1775 & 0.3353 & 0.2143 & 0.8129 & 0.7015 & 0.5267 & 0.3757 \\
\hline & ENG & 0.6358 & 0.4856 & 0.5556 & 0.4034 & 0.6124 & 0.4034 & 0.9316 & 0.8804 & 0.7770 & 0.6534 \\
\hline & IND & 0.7307 & 0.5947 & 0.6602 & 0.5124 & 0.7106 & 0.5704 & 0.9549 & 0.9196 & 0.8441 & 0.7455 \\
\hline \multirow[t]{6}{*}{ Winner } & NZ & 0.5595 & 0.4072 & 0.4764 & 0.3298 & 0.5348 & 0.3834 & 0.9083 & 0.8427 & 0.7172 & 0.5783 \\
\hline & PAK & - & - & 0.5698 & 0.4173 & 0.6260 & 0.4751 & 0.9351 & 0.8863 & 0.7869 & 0.6663 \\
\hline & SA & 0.7208 & 0.5827 & - & - & 0.7003 & 0.5582 & 0.9527 & 0.9159 & 0.8375 & 0.7360 \\
\hline & SR & 0.6713 & 0.5249 & 0.5940 & 0.4418 & - & - & 0.9409 & 0.8960 & 0.8031 & 0.6881 \\
\hline & ZIM & 0.1917 & 0.1137 & 0.1452 & 0.0841 & 0.1767 & 0.1040 & - & - & 0.3213 & 0.2038 \\
\hline & WI & 0.4808 & 0.3337 & 0.3988 & 0.2640 & 0.4560 & 0.3119 & 0.8784 & 0.7962 & - & - \\
\hline
\end{tabular}

AUS: Australia, SA: South Africa, NZ: New Zealand, IND: India, ENG: England, SR: Sri Lanka, PAK: Pakistan, WI: West Indies, BAN: Bangladesh, ZIM: Zimbabwe 


\section{Conclusion}

In this study, we compared the relative performance of ten ODI cricket teams using Bradley-Terry paired comparison model accounting for home-ground effect. Comparison of team performance in ODI cricket using win-loss percentages shows that the higher a team's winning percentage the stronger the team and vice-versa. This provides a team's overall ability but does not quantify the degree to which specific factors such as home and away may have contributed to their performance. A team is considered superior when performs competitively regardless of playing at home or away. On the other hand, a team winning on a consistent basis at home and not performing equally when they play away may be due to the effect of home ground advantage. In some occasions, a weaker home team may perform better and restrict the winning potential of a stronger opponent because of the home ground effect. Therefore, we considered statistical models to study the relative abilities to compete teams based on match outcomes after adjusting for home ground effects.

The Bradley-Terry model considered in this study distinguishes among these teams by calculating individual abilities through the outcomes of the played matches. Teams traditionally perform better at home than away for various reasons, including familiarity with local conditions, the condition of pitches, crowd support, less travel fatigue, among others. In this paper, we have justified that adjustment for home and away venue effect is needed and showed how the winning probabilities differed as a result. Our analysis reveals that most of the team are more likely to win a match at home than away. Some of these teams have probabilities of winning against any particular team calculated greater than 0.5 at home ground, whereas those probabilities are less than 0.5 if they play abroad. Several other factors can be considered to analyze the performance of cricket teams such toss win, the strength of each team, a number of different types of players, player skills, team-wise investment in cricket etc. We keep it as a future scope for analyzing ODI cricket performance data. The interested researcher can consider all ODI cricket playing countries' data to explore their performance.

\section{References}

[1] Abbas, N. and Aslam, M. (2009). Prioritizing the items through paired comparison models, a Bayesian approach. Pakistan journal of Statistics, 25(1):59-59.

[2] Agresti, A. (2014). Categorical data analysis. John Wiley \& Sons.

[3] Allsopp, P. and Clarke, S. R. (2004). Rating teams and analysing outcomes in one day and test cricket. Journal of the Royal Statistical Society: Series A (Statistics in Society), 167(4):657-667.

[4] Bailey, M. and Clarke, S. R. (2006). Predicting the match outcome in one day international cricket matches, while the game is in progress. Journal of sports science \& medicine, 5(4):480-487.

[5] Bradley, R. A. (1953). Some statistical methods in taste testing and quality evaluation. Biometrics, 9(1):22-38. 
[6] Bradley, R. A. and Terry, M. E. (1952). Rank analysis of incomplete block designs: I. the method of paired comparisons. Biometrika, 39(3/4):324-345.

[7] Cattelan, M., Varin, C., and Firth, D. (2010). Stochastic dynamic thurstone-mosteller models for sports tournaments. University of Warwick. Centre for Research in Statistical Methodology.

[8] Cerqueira, P. H., Nakamura, L. R., Pescim, R. R., \& Leandro, R. A. (2017). INVESTIGATING THE UNDERLYING CAUSAL NETWORK ON EUROPEAN FOOTBALL TEAMS. Journal of Data Science, 15(2).

[9] Clarke, S. R. (1988). Dynamic programming in one-day cricket-optimal scoring rates. Journal of the Operational Research Society, 39(4):331-337.

[10] de Silva, B. M. and Swartz, T. B. (1997). Winning the coin toss and the home team advantage in one-day international cricket matches. The New Zealand Statistician, 32(2):16-22.

[11] Glenn, W. and David, H. (1960). Ties in paired-comparison experiments using a modified thurstone-mosteller model. Biometrics, 16(1):86-109.

[12] Gweshe, T. and Durbach, I. (2013). An analysis of the efficiency of player performance at the 2011 cricket world cup. ORiON, 29(2):137-153.

[13] Hallinan, S. E. (2005). Paired comparison models for ranking national soccer teams. $\mathrm{PhD}$ thesis, WORCESTER POLYTECHNIC INSTITUTE.

[14] Henery, R. J. (1992). An extension to the Thurstone-Mosteller model for chess. Journal of the Royal Statistical Society. Series D (The Statistician), 41:559-567.

[15] ICC (2016). Live cricket scores \& news international cricket council. pages Accessed Aug 12, 2016.

[16] Joe, H. (1990). Extended use of paired comparison models, with application to chess rankings. Journal of the Royal Statistical Society. Series C (Applied Statistics), 39(1):85-93.

[17] Lewis, A. (2008). Extending the range of player-performance measures in one-day cricket. Journal of the Operational Research Society, 59(6):729-742.

[18] Louzada, F., Suzuki, A. K., \& Salasar, L. E. (2014). Predicting Match Outcomes in the English Premier League: Which Will Be the Final Rank? Journal of Data Science, 12(2), 235-254.

[19] Neil, D. and Jonathan, G. (2015). Using bradley-terry models to analyse test match cricket. Working papers.

[20] Schauberger, G. and Tutz, G. (2015). Extended ordered paired comparison models with application to football data from german bundesliga. AStA Advances in Statistical Analysis, 99(2):209-227.

[21] Sen, S., Biji, M. S., Chakraborty, S., Sindhu, N. R., \& Balasubramanian, S. (2015). Pairwise Comparison in Repeated Scores-Application in Palliative Cancer Patients. Journal of Data Science, 13(4), 795-806. 
[22] Stern, H. (1990a). A continuum of paired comparisons models. Biometrika, 77:265273.

[23] Stern, H. (1990b). Models for distributions on permutations. Journal of the American Statistical Association, 85:558-564.

[24] Stern, S. E. (2011). Moderated paired comparisons: A generalized bradley-terry for continuous data using a discontinuous penalized likelihood function. Applied Statistics: Journal of the Royal Statistical Society, Series C, 60(3):397-415.

[25] Thurstone, L. L. (1927). A law of comparative judgment. Psychological review, 34(4):273-286.

\author{
Md. Mazharul Islam \\ Institute of Statistical Research and Training, University of Dhaka \\ Dhaka-1000, Bangladesh \\ Jahidur Rahman Khan \\ Institute of Statistical Research and Training, University of Dhaka \\ Dhaka-1000, Bangladesh \\ Enayetur Raheem \\ Biomedical Research Foundation (BRF), Dhaka, Bangladesh.
}

